\title{
Interactions of EGF, Wnt and HOM-C genes specify the P12 neuroectoblast
}

\section{fate in C. elegans}

\author{
Lily I. Jiang and Paul W. Sternberg* \\ Howard Hughes Medical Institute and Division of Biology, California Institute of Technology, Mail Code 156-29, Pasadena, CA \\ 91125, USA \\ *Author for correspondence (e-mail: PWS@cco.caltech.edu)
}

Accepted 3 April; published on WWW 19 May 1998

\section{SUMMARY}

We investigate how temporal and spatial interactions between multiple intercellular and intracellular factors specify the fate of a single cell in Caenorhabditis elegans. P12, which is a ventral cord neuroectoblast, divides postembryonically to generate neurons and a unique epidermal cell. Three classes of proteins are involved in the specification of P12 fate: the LIN-3/LET-23 epidermal growth factor signaling pathway, a Wnt protein LIN-44 and its candidate receptor LIN-17, and a homeotic gene product EGL-5. We show that LIN-3 is an inductive signal sufficient to promote the P12 fate, and the conserved EGF signaling pathway is utilized for P12 fate specification; egl-5 is a downstream target of the lin-3/let-23 pathway in specifying P12 fate; and LIN-44 and LIN-17 act synergistically with lin3 in the specification of the P12 fate. The Wnt pathway may function early in development to regulate the competence of the cells to respond to the LIN-3 inductive signal.

Key words: EGF, HOM-C, Wnt, Cell fate specification, Gene interactions, Ceanorhabditis elegans

\section{INTRODUCTION}

In certain developmental fields, cells are exposed to multiple extrinsic signaling molecules that combine with intrinsic factors to control cell proliferation, differentiation, and morphogenesis. Although we have an increasing understanding of how a single factor regulates cellular behaviors, we have limited understanding of how multiple factors work in concert to ensure cells take on their correct fates. Here we investigate how an epidermal growth factor (EGF)-like protein, a Wnt protein, and a homeotic cluster (HOM-C) transcription factor interact to specify P12 neuroectoblast fate in Caenorhabditis elegans.

The cells P11 and P12 are the most posterior pair of postembryonic ventral cord precursors (Sulston and Horvitz, 1977). These two cells both have the potential to express P12 fate during the early first larval (L1) stage before they enter the ventral cord (Sulston and White, 1980). When either cell is ablated by laser microsurgery, the remaining one takes on P12 fate. How is the P12 fate specified? Though no systematic studies on P11/P12 cell fate specification have been reported, previous investigations have accumulated data indicating that several classes of genes are involved in P11/P12 cell fate specification. These include genes of the lin-3/let-23 pathway, which is the C. elegans EGF signaling pathway, a Wnt family gene lin-44, and a HOM-C gene egl-5.

These three classes of genes have been primarily studied for reasons other than P11/P12 cell fate specification. The lin-3/let23 signaling pathway, which is similar to the EGF signaling pathway of mammals (Fantl et al., 1993), has been studied extensively in $C$. elegans vulval development (reviewed by Sundaram and Han, 1996; Kornfeld, 1997). lin-3 encodes a membrane-spanning protein with a single extracellular EGF domain that is similar in structure to members of the EGF family of growth factors (Hill and Sternberg, 1992). let-23 encodes a receptor tyrosine kinase of the EGF receptor family (Aroian et al., 1990). LIN-3 functions as an intercellular signal and its activity is both necessary and sufficient for vulval induction (Ferguson and Horvitz, 1985; Hill and Sternberg, 1992; Katz et al., 1995), while LET-23 is the likely receptor for the LIN-3 signal. LET-23 transduces LIN-3 activity via an SH2/SH3 adaptor protein SEM-5 (Clark et al., 1992a), which in turn activates the downstream RAS protein LET-60 (Han and Sternberg, 1990). lin-15 defines a general inhibitory pathway that negatively regulates let-23 activity and prevents cells undergoing vulval differentiation (Ferguson and Horvitz, 1985; Ferguson et al., 1987; Herman and Hedgecock, 1990; Clark et al., 1994; Huang et al., 1994). The lin-3/let-23 signaling pathway is also utilized to specify certain cell fates in male spicule development (Chamberlin and Sternberg, 1994).

The C. elegans gene lin-44 is a member of the Wnt family genes (Herman et al., 1995). Wnt family genes encode secreted glycoproteins and are implicated in intercellular signaling to control cell fate specification and cell proliferation in many organisms (reviewed by Nüsse and Varmus, 1992; Moon et al., 1997). In C. elegans, while the major defects in lin-44 mutants appear to be the reversed polarities of certain asymmetric cell divisions in the tail region, they are also defective in the specification of P12 fate (Herman and Horvitz, 1994). Both in 
situ hybridization and the analysis of reporter gene constructs indicated that lin-44 is expressed in epidermal cells at the tip of the tail and posterior to the cells affected by lin-44 mutation (Herman et al., 1995). Mosaic analysis demonstrated that lin44 acts cell nonautonomously and lin-44 function is required in the cells in which lin-44 expression is observed. lin-17 encodes a protein with seven putative transmembrane domains, homologous to the Drosophila Frizzled protein (Sawa et al., 1996). lin-17 regulates several asymmetric cell divisions and cell fates which are also controlled by lin-44 (Sternberg and Horvitz, 1988), therefore has been proposed to be a receptor for the Wnt protein LIN-44 (Sawa et al., 1996).

HOM-C genes are conserved throughout metazoan evolution. These HOM-C transcription factors establish position-specific fates along body axes (Lewis, 1978; reviewed by Krumlauf, 1994). HOM-C genes can act in multiple cell types and at multiple time during development (reviewed by Lawrence and Morata, 1994; Salser and Kenyon, 1994). They are regulated by multiple intercellular and intracellular molecules (Immergluck et al., 1990). They also regulate diverse targets to control position-specific cell fates (Mastick et al., 1995). In C. elegans a cluster of four HOM-C genes has been identified. Along the anteroposterior body axis it consists of lin-39, mab-5, and egl-5, respective homologues of the Drosophila genes Sex combs reduced/deformed/proboscipedia, Antennapedia, and Abdominal-B, with a labial homologue, ceh-13, being between lin-39 and mab-5 (reviewed by Salser et al., 1994). lin-39 activity is required in the central body region for vulval differentiation and patterning, while mab-5 and $e g l-5$ are involved in cell fate specification of the tail region (Kenyon 1986; Chisholm, 1991; Clark et al., 1993; Salser et al., 1993; Clandinin et al., 1997; Maloof and Kenyon, 1998).

We are particularly interested in these three classes of genes because mutations in all these genes show P11/P12 cell fate specification defects. We sought to determine how these intrinsic and extrinsic factors act together to specify a certain cell fate. We first demonstrate that LIN-3 is an inductive signal for the P12 fate, and the lin-3/let-23 pathway functions to specify $\mathrm{P} 12$ fate in a similar way to its function during vulval induction. Then we examine whether the HOM-C gene, egl-5, sets up the competence of the cells to the LIN-3 inductive signal by establishing a pre-pattern, or if it plays an instructive role in P11/P12 cell fate specification. Our results support the latter possibility, that egl-5 is a downstream target of the lin$3 /$ let-23 pathway and its activity is required in executing P12 fate. Finally we explore the interactions between the lin-3/let23 and the Wnt signaling pathways. Our results suggest that lin-44 and lin-3 may act at different times during development corresponding to two genetically parallel pathways, and the Wnt pathway may regulate the competence of the P11/P12 cells to respond to the LIN-3 signal.

\section{MATERIALS AND METHODS}

\section{General methods}

Routine culturing, maintenance and genetic manipulations of $C$. elegans strains were performed according to standard procedures (Brenner, 1974). N2 (Bristol) strain was used as wild type (Brenner, 1974). The following strains were used.

LG I: lin-17(n671) (Ferguson and Horvitz, 1985), lin-44(n1792) (Herman and Horvitz, 1994),
LG II: let-23(sy97) (Aroian and Sternberg, 1991),

LG III: egl-5(n945) (Chisholm, 1991),

LG IV: dpy-20(e1282) (Brenner, 1974), lin-3(n378) (Ferguson and Horvitz, 1985), lin-3(n1059) (Ferguson and Horvitz, 1985), let60(sy93) (Han et al., 1990),

LG X: lin-15(e1763) (Ferguson and Horvitz, 1985), sem-5(n2019) (Clark et al., 1992a),

Transgenic strains:

PS1427: unc-31(e169); syIs6[unc-31(+), pRH51(hsLIN-3EGF) $50 \mathrm{ng} / \mathrm{\mu l}$ (Katz et al., 1995),

PS2467: dpy-20(e1282); syEx178[dpy-20(+), pLJ5(hsEGL-5) $20 \mathrm{ng} / \mu \mathrm{ll}$,

PS2347: dpy-20(e1282); muIs13[rol-6(su1006), egl-5-lacZ] him5(e1490) (Wang et al., 1993).

\section{Construction of hsp16.1-egl-5 transgene}

The hsp16.1-egl-5 construct, pLJ5, was constructed in the same way as previously described constructs $h s-m a b-5$ and $h s$-lin-39 (Salser and Kenyon, 1992; Hunter and Kenyon, 1995). Briefly, primers overlapping sequences of the start and stop codons were used to amplify the coding sequence of egl-5 from an egl-5 cDNA clone by PCR. The amplification products were sequenced and ligated into the HpaI site of the hsp16.1 gene (Russnak and Candido, 1985). This construct generates a fusion protein with the first 44 amino acids of hsp16.1 gene product and the full length EGL-5 protein.

\section{Construction of transgenic strains}

Transgenic animals were generated by microinjection of DNA into the mitotic germline of hermaphrodites according to the method of Mello et al. (1991). syEx178 was obtained by microinjection of pLJ5 plasmid at $20 \mathrm{ng} / \mu \mathrm{l}, \mathrm{pMH} 86(\mathrm{dpy}-20(+))$ at $15 \mathrm{ng} / \mu \mathrm{l}$, and carrier DNA (pBlueScript) at $120 \mathrm{ng} / \mu \mathrm{l}$ into $d p y-20(e 1282)$ mutant animals. To test the function of the construct, the transgenic line syEx178 was crossed into egl-5(n945); dpy-20(e1282) and let-23(sy97) unc4(e120)/mnCl(dpy-10 unc-52); dpy-20(e1282) strains. The non-Dpy phenotype indicates the presence of the transgene.

syIs 6 was obtained by X-ray integration of the syEx23 strain, which expresses the EGF domain of LIN-3 under the heat shock promoter and has been described by Katz et al. (1995).

muIs13 is an integrated strain containing the egl-5-lacZ transgene (Wang et al., 1993), kindly provided by Craig Hunter. For ease of scoring, dpy-20(e1282) mutation was crossed into the strain to suppress the Roller phenotype caused by the transformation marker.

syIs6; dpy-20(e1282); muIs13 him-5(e1490) strain was constructed by crossing syIs6; dpy-20(e1282); him-5(e1490) males with muIs 13 him-5(e1490) hermaphrodites. Animals homozygous for syIs6 and muIs 13 displayed 100\% multivulva phenotype under heat shock condition and had $100 \% \beta$-galactosidase-positive staining.

The assay for $\beta$-galactosidase activity was performed as described previously (Fire et al., 1990).

\section{Heat shock of transgenic animals}

Cohorts of adult hermaphrodites were placed on standard worm culture plates to collect eggs. The hermaphrodites were transferred to a fresh plates every hour. The age of the worms at heat shock was determined as hours after egg-lay. Different strains hatch at slightly different times ( \pm 2 hours after egg-lay), and times were normalized to hatching. Animals were heat shocked in a $33^{\circ} \mathrm{C}$ water bath for 30 minutes or 60 minutes as indicated in each figure legend. For the experiment involving EGL-5 overexpression in a let-23(sy97) mutant background, eggs were collected by cutting egg-laying defective adult hermaphrodites into halves and then heat shocking at certain developmental times.

\section{Anatomical and cell lineage analysis}

L3 or early-mid L4 hermaphrodites were examined with Nomarski optics. P11 and P12 fates were determined according to the distinct nuclear morphologies and positions of P11.p and P12.pa cells. Cell 
lineages were followed at $20^{\circ} \mathrm{C}$ as described by Sulston and Horvitz (1977).

\section{RESULTS}

\section{P11/P12 development}

In $C$. elegans there are twelve ventral cord precursor cells, P1$\mathrm{P} 12$, numbered from anterior to posterior along the body axis. These cells divide postembryonically to generate cells of the ventral nervous system, as well as the vulva (Sulston and Horvitz, 1977). P11/P12 are the most posterior pair of the ventral cord precursors. At hatching, the cells AB.plapappa (left side) and AB.prapappa (right side) are disposed laterally (Sulston et al., 1983). In hermaphrodites, they start to migrate ventrally several hours after hatching and enter the ventral cord about 8-9 hours after hatching at $20^{\circ} \mathrm{C}$ (Fig. 1A). The left cell migrates anteriorly and becomes P11, whereas the right cell migrates posteriorly and becomes P12. Two hours later they both divide once. The anterior daughters, P11.a and P12.a are neuroblasts that will divide for three more rounds to generate several ventral cord neurons. These neurons are morphologically indistinguishable under Nomarski optics. The posterior daughter of P11, P11.p, does

Fig. 1. P11/P12 phenotype

observed in Nomarski

photomicrographs. (A) Wild-type

$\mathrm{P} 11 / \mathrm{P} 12$ lineages at $20^{\circ} \mathrm{C}$ (Sulston and Horvitz, 1977). Terminal fates are: $\bullet$, ventral cord neuron; $\mathrm{x}$, programmed cell death. Timing is indicated as AEL, denoting hours after egg-lay, v.c. denotes ventral cord. (B) Wild type, (C) let60(sy93dn), (D) syIs6, (E) wildtype animal, P12.pp undergoes cell death, indicated by a concave arrowhead. (F) P11 and P12 lineages are followed after heat shock treatment of syIs 6 strain $(n=4)$. P11.p divides as does P12.p, and both P11.pp and P12.pp die. (G) $s y E x 178$. In B-D and G, arrowhead indicates P11.p, arrow points to P12.pa. Scale bars, $20 \mu \mathrm{m}$. not divide but rather fuses with the large epidermal syncytium hyp7. P12.p divides once more about 1 hour prior to L1 molt to generate P12.pa, which becomes a unique epidermal cell, hyp12, and P12.pp, which undergoes cell death. P11.p and P12.pa can be distinguished by their different nuclear morphologies and positions observed with Nomarski optics (Fig. 1B). Sulston and White (1980) demonstrated that prior to
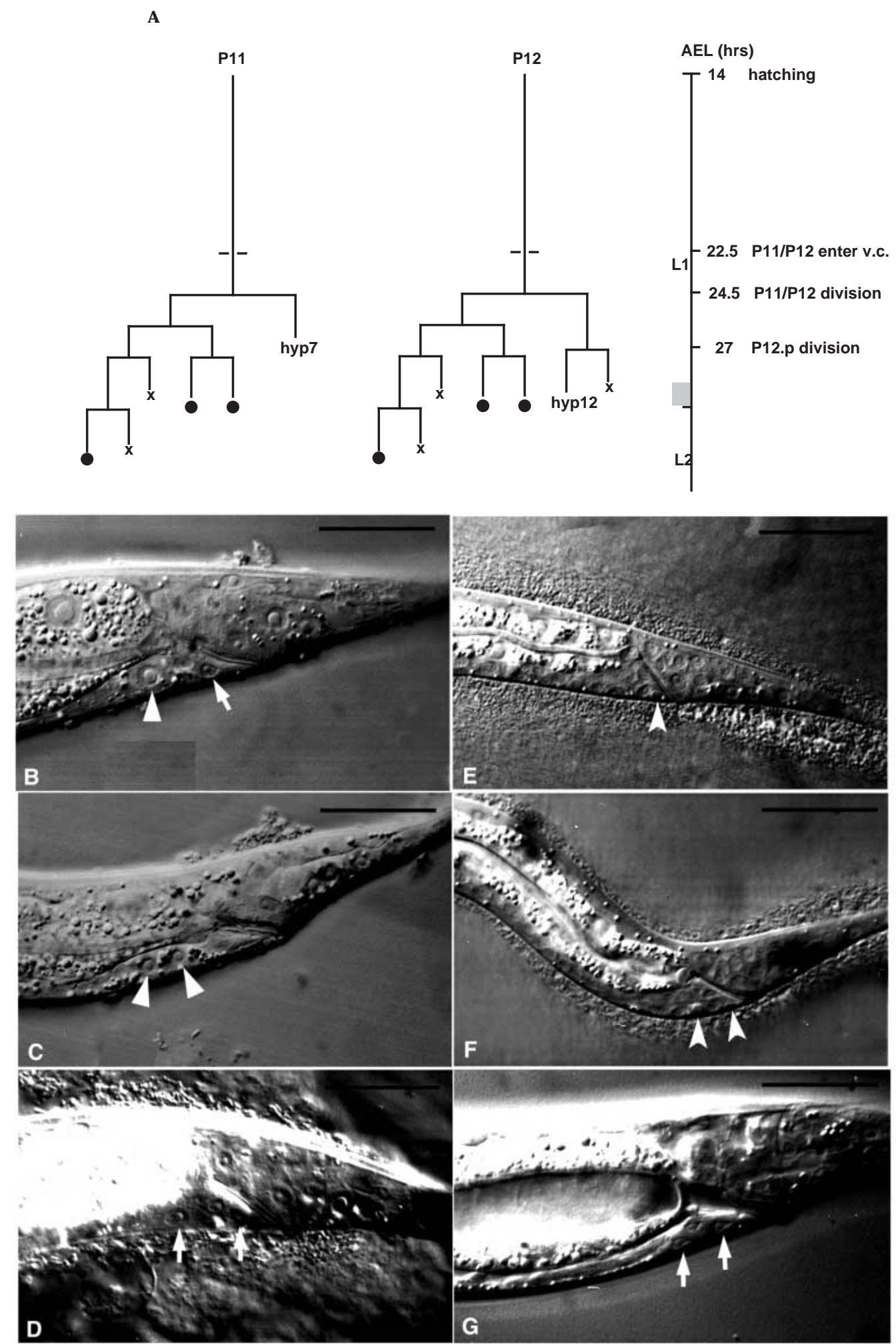
migration both $\mathrm{P} 11$ and $\mathrm{P} 12$ cells are able to express the P12 fate; and if only a single cell is present, it will adopt the P12like fate. Therefore P12 represents a primary fate, while P11 is a secondary fate.

\section{Mutants of several lin-3/let-23 pathway components have defects in P11/P12 cell fate specification}

Mutants of several lin-3/let-23 pathway components have been reported to display defects in P11/P12 cell fate specification. Loss-of-function alleles of let-23 showed a loss of the cell P12.pa with concommitant duplication of P11.p in the hermaphrodite tail (Fixsen et al., 1985; Aroian and Sternberg, 1991). Lineage analysis in males indicated that this defect likely represents a transformation of P12 to P11 fate, as the anterior branch is also affected (Fixsen et al., 1985). Mutations at the lin-15 locus, which encodes negative regulators of the lin-3/let-23 pathway, have the opposite defect, P11 to P12 cell fate transformation (Fixsen et al., 1985). Other components, sem-5 and let-60, which encode a $\mathrm{SH} / \mathrm{SH} 3$ domain protein (Clark et al., 1992a) and a RAS protein (Han and Sternberg, 1990), respectively, are also involved in P11/P12 cell fate specification (Clark et al., 1992b). We examined hermaphrodites homozygous for a reduction-of-function allele of sem-5, n2019, and found a $31 \%$ penetrant P12 to P11 cell fate transformation (Table 1A). A dominant-negative allele of let-60, sy93, also displays a $45 \%$ penetrant $\mathrm{P} 12$ to $\mathrm{P} 11$ fate transformation (Fig. 1C, Table 1A).

Although mutations in these lin-3/let-23 pathway components result in a P11/P12 defect, the penetrance of this phenotype is incomplete. We propose two possible models. The lin-3/let-23 pathway functions in additional capacities including vulval induction, male spicule development, hermaphrodite fertility, and L1 lethality (Aroian and Sternberg, 1991). Although functions such as vulval induction, male spicule development, and hermaphrodite fertility can be genetically separated (Aroian et al., 1994; Lesa and Sternberg, 1997), mutations with P11/P12 defect share a common L1 lethality phenotype. It is possible that the P11/P12 defect and the lethality phenotype require similar aspects of gene function (e.g., expression during the L1 stage), therefore the dead larvae with P11/P12 defect are excluded from scoring. Alternatively, there might exist another pathway acting in conjunction with the lin-3/let-23 pathway for P11/P12 fate specification.

Given the fact that these lin-3/let-23 pathway components are involved in P11/P12 cell fate specification, we asked if the genetic interactions among these genes are conserved. During vulval development, lin-15 defines a general inhibitory pathway which negatively regulates let-23 activity. As let-23 and lin-15 also have an opposite effect on P11/P12, we tested if lin-15 acts at the level of let-23. We constructed a strain defective in both genes. $30 \%$ of let-23; lin-15 double mutants display a P12 to P11 cell fate transformation (Table 1A), resembling the mutation in let-23 alone. Thus let-23 is epistatic to lin-15, reminiscent of lin-15 negative regulation of let-23 in vulval induction.

\section{LIN-3 is an inductive signal sufficient for P12 fate}

Since none of the existing lin-3 mutants exhibits any defect in P11/P12 cell fate specification and null alleles of lin-3 are L1 lethal, it has been difficult to determine if lin-3 plays a role in P11/P12 cell fate specification. We circumvented this problem by testing whether overexpression of LIN-3 would affect P11

Table 1.

\begin{tabular}{|c|c|c|c|c|}
\hline $\begin{array}{l}\text { A. let- } 23 \text { pathway is inv } \\
\text { Genotype* }\end{array}$ & \multicolumn{3}{|c|}{ A. let-23 pathway is involved in $\mathrm{P} 11 / \mathrm{P} 12$ cell fate specification. } & \\
\hline N2 (wild type) & 50 & 0 & 0 & \\
\hline let-23(lf) & 50 & 0 & 44 & \\
\hline lin-15(lf) & 74 & 54 & 0 & \\
\hline let-23(lf); lin-15(lf) & 49 & 0 & 33 & \\
\hline $\operatorname{sem}-5(\mathrm{lf})$ & 45 & 0 & 31 & \\
\hline let-60(dn) & 51 & 0 & 45 & \\
\hline \multicolumn{5}{|c|}{ B. egl-5 is epistatic to the lin-3/let-23 pathway in $\mathrm{P} 12$ fate specification. } \\
\hline Genotype* & $n \dagger$ & $\% \mathrm{P} 11 \rightarrow \mathrm{P} 12 \ddagger$ & $\%$ wild type & $\% \mathrm{P} 12 \rightarrow \mathrm{P} 11 \S$ \\
\hline$e g l-5(l f)$ & 63 & 0 & 0 & 100 \\
\hline egl-5(lf); lin-15(lf) & 41 & 0 & 0 & 100 \\
\hline egl-5(lf); let-23(lf) & 25 & 0 & 0 & 100 \\
\hline syIs6, non-hs & 50 & 0 & 100 & 0 \\
\hline egl-5(lf); syIs6, non-hs & 50 & 0 & 0 & 100 \\
\hline egl-5(lf); syIs6], $h s$ & 37 & 0 & 0 & 100 \\
\hline let-23(lf) & 50 & $0 * *$ & $56+\dagger$ & $44+\ddagger$ \\
\hline let-23(lf); syEx178, hs & 21 & $15 * *$ & $75 \dagger \dagger$ & $10+\ddagger$ \\
\hline
\end{tabular}

non-hs, animals were not treated with heat shock; hs, animals were heat shocked.

*Alleles used are: let-23(sy97), a severe reduction-of-function allele; lin-15(e1763), a severe reduction-of-function allele, sem-5(n2019), a reduction-offunction allele; let-60(sy93), a dominant-negative allele; egl-5(n945), a severe reduction-of-function. syIs6 is a transgenic strain bearing the $h s L I N-3 E G F$ construct, $s y E x 178$ is a transgenic strain bearing the $h s E G L-5$ construct.

$\dagger$ Number of animals examined.

\$Percentage of animals with two P12-like cells. Animals were scored under Nomarski at L3-L4 stage, cell fate transformation is determined by nuclear morphology and position.

$\S$ Percentage of animals with two P11-like cells.

IIAnimals were heat shocked during embryogenesis and L1 stage in $33^{\circ} \mathrm{C}$ water bath for 30 minutes.

$* * P=0.0223$, Fisher's Exact Test

$\dagger \dagger P=0.7935$, Fisher's Exact Test

$\$+P=0.0057$, Fisher's Exact Test 
and P12. A transgenic strain bearing a construct that expresses the EGF domain of LIN-3 under the control of a heat shock promoter/enhancer was used for this experiment. This transgene, the 63 amino acid EGF domain of LIN-3 driven by the inducible and tissue general hspl641 heat shock promoter (Stringham et al., 1992), has been demonstrated to be functional and sufficient to induce vulval development (Katz et al., 1995). The transgenic strain syIs6 carries a high dose of the hslin-3EGF plasmid integrated in the genome. When these transgenic animals are grown at $25^{\circ} \mathrm{C}$, the basal activity of LIN-3EGF can induce a multivulva phenotype, whereas the P11 and P12 cells remain wild type. We carried out a time course study to induce the expression of LIN-3EGF during embryogenesis and the L1 stage by a pulse of heat shock treatment at certain developmental times (Fig. 2A). We first observed that overexpression of LIN-3EGF does lead to a duplication of the cell P12.pa with a concommitant loss of P11.p in the hermaphrodite tail (Fig. 1D). We interpret this as a gain-offunction lin-3 phenotype by analogy with results from the vulva and the spicules (Katz et al., 1995; Chamberlin and Sternberg, 1994). We confirmed this phenotype as being the result of the $\mathrm{P} 11$ to $\mathrm{P} 12$ cell fate transformation by following the P11/P12 lineages in hermaphrodites after heat shock $(n=4)$. As shown in Fig. 1F, P11.p behaves like P12.p: it divides an extra round, and P11.pp undergoes cell death as does P12.pp. Also, the P11 to P12 cell fate transformation occurs when overexpression of LIN-3EGF is induced at late embryogenesis; it peaks to almost $100 \%$ when induced within several hours after hatching. Later, the effect of LIN-3EGF tapers off; overexpression of LIN-3EGF does not have any effect on $\mathrm{P} 11 / \mathrm{P} 12$ cell fate after the P11/P12 cells enter the ventral cord. This timing is consistent with the ablation results of Sulston and White (1980) who found that the P11 cell loses its competence to become P12 after it enters the ventral cord. We conclude that LIN-3 is sufficient to induce the P12 fate and infer that LIN-3 is an inductive signal for the $\mathrm{P} 12$ fate.

\section{egl-5 acts in a common pathway with the lin-3/let-23 pathway in P12 fate specification}

The C. elegans HOM-C gene egl-5 is a homolog of the Drosophila Abdominalrespectively.
$B$ gene and is important for patterning the tail region of $C$. elegans (Chisholm, 1991; Wang et al., 1993). Loss-of-function mutations in egl-5 cause a P12 to P11 cell fate transformation, indicating that egl-5 activity is required for the P12 fate (Kenyon,



Fig. 2. Time course of the effect of overexpression of LIN-3EGF and EGL-5 on P11/P12 fate specification. (A) syIs6(hsLIN-3EGF). (B) syIs6(hsLIN-3EGF); lin-44(n1792).

(C) $s y E x 178(h s E G L-5)$. Eggs were collected every hour on fresh plates and heat shocked at certain times after egg-lay as indicated on the abscissa, AEL denotes after-egg-lay. The heat shock conditions were $33^{\circ} \mathrm{C}, 30$ minutes for $\mathrm{A}$ and $\mathrm{B}$, and $33^{\circ} \mathrm{C}, 60$ minutes for $\mathrm{C}$. Open bars indicate percentage of $\mathrm{P} 11$ to $\mathrm{P} 12$ transformation; hatched bars indicate $\mathrm{P} 12$ to $\mathrm{P} 11$ transformation. Error bars are $95 \%$ confidence limits based upon the binomial distribution. Dashed lines indicate the times of hatching, P11/P12 cells entering the ventral cord, and the first division of P11/P12 cells, which are about 14, 22.5, and 27 hours after egg-lay 
1986; Chisholm, 1991). We examined the epistasis relationship between egl-5 and mutations of the lin-3/let-23 pathway. Doubly mutant strains were constructed bearing an egl-5 mutation and several lin-3/let-23 pathway components. egl-5(n945) mutants display a $100 \%$ penetrance of P12 to P11 transformation. All the double mutants behave like mutation in egl-5 alone (Table 1B). For example, $l i n-15(l f)$ animals display a $54 \%$ penetrance of P11 to P12 fate transformation, while all egl-5(lf); lin-15(lf) double mutants show a P12 to P11 fate transformation. Moreover, when overexpression of LIN-3EGF is induced in egl-5 mutant animals, its effect on P11/P12 is completely blocked by egl-5 mutation (Table 1B). Thus egl-5 acts in a common pathway with the lin3/let-23 pathway in P12 fate specification.

\section{egl-5 plays an instructive rather than permissive role in P12 fate specification}

Previous studies have shown that HOM-C genes can specify the competence of cells to respond to an intercellular signal, thus establishing a pre-pattern in a developmental field (Clandinin et al., 1997). They themselves can also be regulated by intercellular signaling molecules (e.g., Immergluck et al., 1990; Maloof and Kenyon, 1998). egl-5 might play a permissive role in P12 fate specification by setting up the competence of the cell to respond to the LIN-3 inductive signal, or egl-5 might be an instructive factor for $\mathrm{P} 12$ fate specification. To distinguish these hypotheses, we first tested if overexpression of EGL-5 has an effect on P11/P12 cell fate specification. We engineered the full length egl-5 cDNA (Salser and Kenyon, 1992) under the control of the hspl6.1 promoter. Transgenic animals bearing this $\mathrm{pLJ} 5$ construct as an extrachromosomal array, syEx 178 , were generated. Upon heat shock treatment, the hspl6.1 promoter should drive EGL-5 expression ubiquitously in somatic tissues (Russnak and Candido, 1985). When syEx178 was introduced into egl-5(n945) mutants, expression of the transgene was able to rescue the P11/P12 mutant phenotype (data not shown). Overexpression of EGL5 has a dominant sterile phenotype (L. Jiang, unpublished observation), making it impossible to examine the rescue of the egg-laying behavior. To analyze the effect of EGL-5 in wild-type animals, a pulse of EGL-5 expression was induced in the transgenic strain during late embryogenesis and L1 stage (Fig. 2C). Overexpression of EGL-5 in wildtype animals does lead to a P11 to P12 fate transformation (Fig. 1G). The most effective time to cause a P11 to P12 fate transformation is when overexpression of EGL-5 is induced around the time of the entry of P11/P12 into the ventral cord. This is later than the time of maximal effect of LIN-3EGF overexpression, which is within several hours after hatching. This temporal difference in responsiveness is consistent with our observation that egl-5 is downstream of the lin-3/let-23 pathway. It is also consistent with the observation that egl-5 is not expressed in P cells during the early L1 stage (Wang et al., 1993).

We then asked if overexpression of EGL-5 could suppress the P11/P12 defect of let-23 mutants. If egl5 is a permissive factor for $\mathrm{P} 12$ fate, no rescue of the P11/P12 defect would be expected; whereas if egl-5 is an instructive factor, overexpression of EGL-5 should be able to rescue the P11/P12 defect caused by let-23 mutation, and may additionally result in a P11 to P12 cell fate transformation. $44 \%$ of let-23(sy97) animals show a P12 to P11 transformation and 56\% are wild type. When overexpression of EGL-5 is induced in transgenic let-23(sy97) animals bearing syEx178, the percentage of animals with a P12 to P11 transformation is lowered to $10 \%(P=0.0057$, Fisher's Exact Test), while the percentage of wild-type animals is increased to $75 \%$, the remaining $15 \%$ animals display a P11 to P12 cell fate transformation $(P=0.0223)$ (Table 1B). This result suggests that overexpression of EGL-5 suppresses the P11/P12 defect of let-23 mutants, as predicted by the instructive model. Therefore egl-5 plays an active role in P12 fate specification and acts downstream of let-23.

The effect of overexpression of EGL-5 in a let-23 deficient background is not as prominent as it is in a wild-type background. This may be attributed to technical limitation: let23(sy97) worms are egg-laying defective so that mixed stage animals were collected for the heat shock experiments and timing may have been imprecise. Another possibility is that there exists a feedback loop between egl-5 and the lin-3/let-23 pathway.

\section{egl-5 is a downstream target of the lin-3/let-23 pathway in specifying P12 fate}

We next tested whether egl-5 is a downstream target of the lin3/let-23 pathway for P12 fate specification. Expression of an egl-5-lac Z reporter gene is detected in the cell P12.pa. As overexpression of LIN-3EGF leads to a P11 to P12 cell fate transformation, we examined if the transformed P11.pa cell is

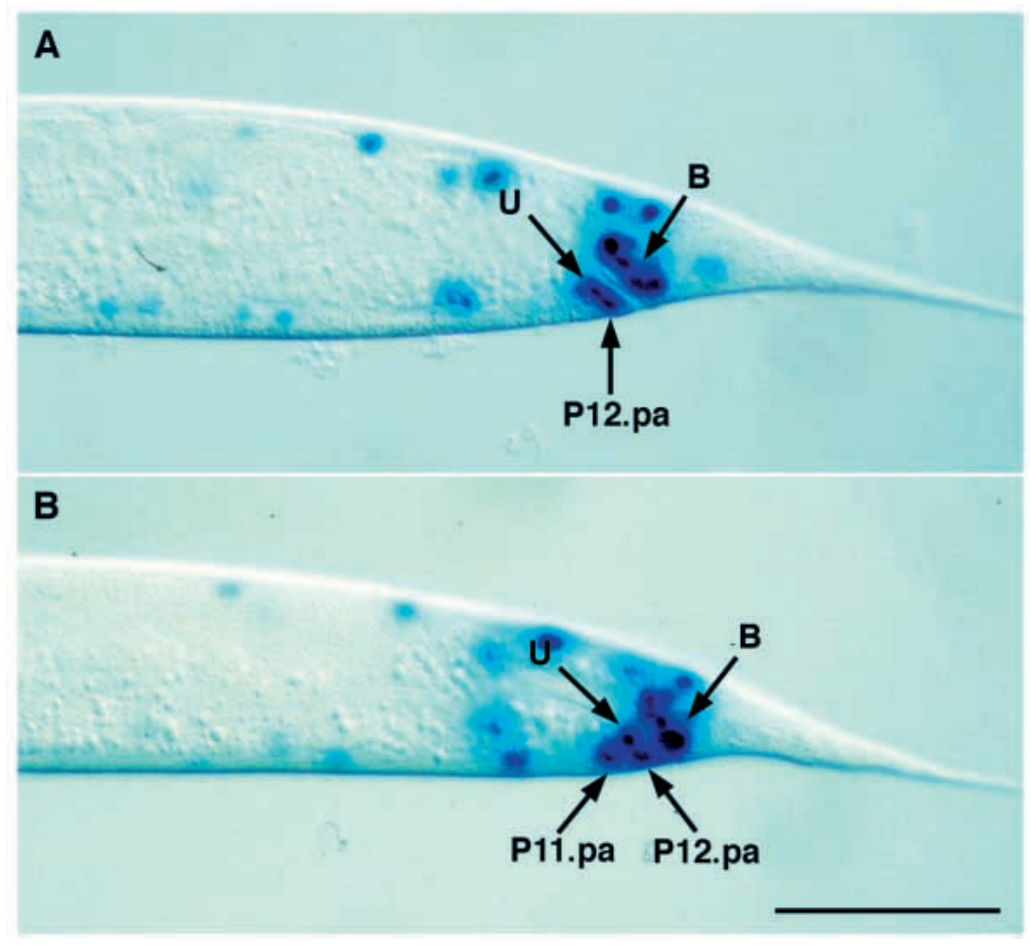

Fig. 3. LIN-3 can induce egl-5 transcription in P12 lineage. (A) In wild-type animals, egl-5-lacZ expression is detected in P12.pa cell. (B) When overexpression of LIN-3EGF induces P11 to P12 transformation, egl-5-lacZ expression is detected in both P11.pa and P12.pa cells. Scale bar, $20 \mu \mathrm{m}$. 
able to express the egl-5-lacZ marker. A strain bearing both the $h$ s-lin-3EGF and the egl-5-lacZ transgenes was constructed. When overexpression of LIN-3EGF is induced at the proper time based on results of Fig. 2A, which is 15-17 hours after eggs were laid, a P11 to P12 cell fate transformation occurs in about $70 \%$ animals. As shown in Fig. 3, egl-5-lacZ expression is turned on in both the normal P12.pa and the transformed P11.pa cells. This observation indicates that egl-5 expression in P12.pa can be turned on upon activation of the lin-3/let-23 pathway.

\section{Does lin-44 act via lin-3?}

lin-44, a gene encoding a Wnt family protein (Herman et al., 1995), is a member of a third class of genes that affect P11/P12 cell fate specification (Herman and Horvitz, 1994). 16\% of lin44(n1792) mutant animals show a P12 to P11 cell fate transformation. We wondered how the two signaling molecules, LIN-3 and LIN-44, act together to specify P12 fate. One possibility is that lin-44 regulates lin-3 expression by controlling the identity of the cell that normally expresses LIN3 in the tail region. There are several reasons for this hypothesis. First, several cells in the tail region are defective in lin-44 mutants. These include B, F, U, and T cells, which are posterior to the $\mathrm{P} 11 / \mathrm{P} 12$ cells and anterior to lin-44 expressing cells hyp8-11 (Herman et al., 1995). Second, analysis of lin-3-lacZ reporter constructs suggests that a potential source of LIN-3 signal exists in the tail region posterior to the P11/P12 cells (R. Hill, C. Chang and P. Sternberg, unpublished observation). Third, it has been suggested that Wnt genes are involved primarily in short-range cell interactions (van den Heuvel et al., 1989; Vincent and Lawrence, 1994). P11 and P12 lie anterior to the cells affected by lin-44, and may not be in direct contact with the lin-44 expressing cells. Fourth, lin-44 expression is turned on during embryogenesis, which is prior to lin-3 expression in the tail.

If lin-44 only regulates lin-3 expression, overexpression of LIN-3EGF should be able to rescue the P11/P12 defect in lin44 mutants and have the same effect as it does in wild-type background. To test this hypothesis we crossed the $s y I s 6$ strain into lin-44(n1792) mutant background and conducted heat shock experiments. As shown in Fig. 2B, overexpression of LIN-3EGF at early L1 stage rescues the P12 to P11 transformation caused by loss of lin-44 function and results in a P11 to P12 transformation. However, the effect of LIN-3EGF overexpression is not as robust as it is in a wild-type background. In a lin-44 mutant background, overexpression of LIN-3EGF only results in a maximum 50\% P11 to P12 transformation, whereas in a wild-type background the transformation is almost $100 \%$ at the same stage. This difference is not expected if lin-3 expression is solely regulated by lin-44 in the tail region. However, we cannot rule out the possibility that the cells that provide functional LIN-3 might be altered in lin-44 mutants, and thus overexpression of LIN-3EGF in lin-44 mutants might not be as effective as it is in wild type.

To further test the hypothesis that lin-44 regulates lin-3, we did the following two experiments. First, we examined the expression pattern of a lin-3-lacZ reporter gene in a lin-44 mutant background. Transgenic animals bearing a lin-3-lacZ reporter construct which contains $12 \mathrm{~kb}$ of lin-3 genomic sequence have been generated (R. Hill, C. Chang, and P. Sternberg, unpublished observations). lin-3-lacZ is expressed in the hermaphrodite tail region in newly hatched larvae. We believe the expression resides in the cells $\mathrm{K}, \mathrm{K}^{\prime}$, and the daughters of $K$, which are posterior to the P11/P12 cells. When the transgenic strain is crossed into the lin-44(n1792) mutant background, we did not observe any change in the level of expression nor of the position of the cells $(n=45)$.

Second, we examined the P11/P12 phenotype in lin-44; lin15 double mutant animals. lin-15 is a negative regulator of the lin-3/let-23 pathway and acts at the level of let-23 in vulval development (Huang et al., 1994). lin-15(e1763) mutant animals display a $54 \%$ penetrance of P11 to P12 fate transformation (Table 1A). However lin-15 acts in a signaldependent manner as loss of LIN-3 activity removes its effect on P11 and P12 cells. lin-3(n378/n1059) mutants do not display a P11/P12 defect (Table 2), neither do lin3(n378/n1059); lin-15(e1763) double mutant animals ( $n=45)$. If lin-44 solely regulates lin-3 activity, we would expect a similar result. Yet double mutant animals defective in both lin44 and lin-15 show a mixed cell fate transformation, $10 \%$ P12 to P11 fate transformation and $35 \%$ P11 to P12 fate transformation $(n=116)$. This cannot result from a quantitative effect since the lin-44 genotype has a more severe effect on P12 than the lin-3 genotype used. This additive effect argues against the hypothesis that lin-44 affects P11/P12 fate by regulating lin-3 activity. An additional lin-44 function is required for proper $\mathrm{P} 11 / \mathrm{P} 12$ fate specification.

\section{lin-3 and lin-44 act synergistically in P12 fate specification}

We next tested the interactions between lin-3 and lin-44 by examining the P11/P12 defect in a strain defective in both genes. Heterozygotes of a lin-3 reduction-of-function allele, $n 378$, in trans to a null allele, $n 1059$, do not display the P11/P12 defect, but have almost no vulval induction and severely defective spicules. However, $n 378 / n 1059$ enhances the phenotype of lin44(n1792) from $16 \% \mathrm{P} 12$ to $\mathrm{P} 11$ transformation to $78 \%$ $(P<0.0001)$ (Table 2). Although it does not rule out a sequential model where lin-3 acts downstream of lin-44, the strong synergy between lin-3 and lin-44 is consistent with the two signals acting in parallel. To confirm the interaction between lin-3 and lin-44, we tested if synergy exists between lin-3 and lin-17 mutations. lin-17, which encodes a putative seven-transmembrane protein similar to the Drosophila Frizzled protein, has been suggested to be a receptor for the LIN-44 protein (Sawa et al., 1996). lin17(n671) animals have a penetrance of $29 \%$ P12 to P11 fate transformation (Table 2). Double mutant animals defective in both $\operatorname{lin}-17$ and lin-44 display a similar defect to $\operatorname{lin}-17(n 671)$ alone, showing $28 \% \mathrm{P} 12$ to P11 fate transformation $(P=1)$ (Table 2). This lack of synergy supports the hypothesis that LIN-44 signal acts through the receptor LIN-17 for P11/P12 fate specification. We contructed a strain defective in both lin-17 and lin-3. 82\% of lin-17; lin-3 double mutants show P12 to P11 cell fate transformation, resembling lin-44; lin-3 double mutants $(P=0.544)$, while different from lin-17 single mutants $(P<0.0001)$. Therefore, similar synergistic interactions exist between lin-3 and lin-17 as between lin-3 and lin-44. A synergistic interaction is also found between mutations of let-23, the EGF receptor for LIN-3 signal, and the Wnt signal LIN-44 (Table 2). Our data support the hypothesis that both of the two signaling pathways, lin-3 and lin-44, are required for the P12 fate specification. 
Table 2. Synergistic interactions between the lin-3/let-23 and the Wnt signaling pathways

\begin{tabular}{|c|c|c|}
\hline Genotype & $n$ & $\% \mathrm{P} 12 \rightarrow \mathrm{P} 11$ \\
\hline $\operatorname{lin}-3(n 378 / n 1059)^{*}$ & 24 & 0 \\
\hline let-23(sy97) & 50 & 44 \\
\hline $\operatorname{lin}-44(n 1792)$ & 244 & $16 \neq$ \\
\hline $\operatorname{lin}-17(n 671)$ & 41 & $29 \S, \Phi$ \\
\hline lin-17(n671) lin-44(n1792) & 54 & $28 \S$ \\
\hline lin-44(n1792); lin-3(n378/n1059)† & 159 & $78 \$$ \\
\hline $\operatorname{lin}-17(n 671) ; \operatorname{lin}-3(n 378 / n 1059) \dagger$ & 114 & $82 \pi$ \\
\hline lin-44(n1792); let-23(sy97) & 91 & $93 * *$ \\
\hline
\end{tabular}

*A strain of genotype + let-312(s1234)lin-3(n378) + unc-22(s7)/unc$24(e 138)+$ lin-3(n1059) dpy-20(e1282) + was used to examine the P11/P12 phenotype at L3-L4 stage. Homozygotes of lin-3(n1059) are L1 lethal. Homozygotes of lin-3(n378) control display no P11/P12 defect.

$\dagger$ Full genotype of lin-3 region is + lin-3(n378) let-59(s49) + unc$22(s 7) / u n c-24(e 138)$ lin-3(n1059) + dpy-20(e1282) +. As homozygotes of either lin-3(n1059) or let-59(s49) are L1 lethal, only transheterozygous animals bearing lin-3(n378/n1059) would be viable therefore scored at L3-L4 stage.

$\ddagger P<0.0001$, Fisher's Exact Test

$\S P=1$, Fisher's Exact Test

II $P<0.0001$, Fisher's Exact Test

$* * P<0.0001$, Fisher's Exact Test, compared with additive effect which would be $53 \%$.

\section{Bi-directional P11/P12 cell fate transformation}

During the course of our experiments, we found the surprising phenomenon that in two cases, overexpression of the same protein, EGL-5 or LIN-3, can have opposite effect on P11/P12 fate depending on the time of induction. Overexpression of EGL-5 induced in newly hatched larvae causes a P12 to P11 fate transformation (Fig. 2C), which contradicts its role in specifying P12 fate. Overexpression of LIN-3EGF during late embryogenesis potentiates the P12 to P11 cell fate transformation in a lin-44(n1792) background (Fig. 2B). n1792 animals only exhibits $16 \%$ penetrance of the P12 to P11 fate transformation. When overexpression of LIN-3EGF is induced within several hours before hatching in a lin-44 mutant background, the P12 to P11 fate transformation phenotype is increased to $70 \%$. This bi-phasic profile does not occur when overexpression of LIN-3EGF is induced in a wild-type background (Fig. 2A) nor when lin-44(n1792) animals are treated with heat shock at the same stage (data not shown). This surprising phenomenon suggests to us two possibilities. First, the onset of egl-5 expression might be crucial for proper P12 fate specification. Second, the unique effect of LIN-3 overexpression in lin-44 mutant background suggests a requirement of lin-44 function during late embryogenesis for P12 fate specification.

\section{DISCUSSION}

By studying the specification of the P11 and P12 cell fates in C. elegans, we address the question how a cell integrates multiple intrinsic and extrinsic factors to take on its correct fate. We considered three classes of genes: EGF signaling pathway genes, Wnt signaling pathway genes, and a HOM-C gene, which are involved in the P12 neuroectoblast fate specification. Our results support three conclusions. First, LIN3 is an inductive signal for P12 fate and the lin-3/let-23 EGF signaling pathway is required for P12 fate specification. Second, the HOM-C gene egl-5 is a downstream target of the lin-3/let-23 pathway in executing P12 fate. Third, the Wnt gene lin-44 acts in parallel with the LIN-3 signal but at a different developmental time, and the Wnt pathway may regulate the competence of cells to respond to the LIN-3 inductive signal.

\section{The conserved EGF signaling pathway is used to organize cell fate specification in the $C$. elegans tail region}

Previous studies have indicated that mutations in several genes of the lin-3/let-23 pathway displayed P11/P12 cell fate specification defects (Fixsen et al., 1985; Aroian and Sternberg, 1991; Clark et al., 1992b). The genes let-23, lin-15, sem-5, and let-60 are all involved in P11/P12 cell fate specification. We provide evidence that LIN-3 is an inductive signal for P12 fate. First, overexpression of the EGF domain of LIN-3 leads to P11 to P12 cell fate transformation. Second, loss-of-function mutations of lin-3 decrease P12 fate specification to the reverse transformation in a sensitized lin44 mutant background. This is the first demonstration that mutation of lin-3 does have an effect on P12 fate specification, and therefore the effect of LIN-3EGF overexpression is not simply mimicking the effect of another EGF-like protein. Our



Fig. 4. Model for P12 fate specification. Inductive signal LIN-3 activates LET-23 receptor, which activates LET-60 RAS activity and turns on egl-5 expression. Expression of egl-5 at proper time specifies the cell to take on P12 fate. lin-15 negatively regulates let23 activity and prevents the cell becoming P12. Wnt pathway may function at early developmental stage, LIN-44 signal acts via receptor LIN-17 to set up the competence of P11 and P12 cells to respond to the inductive signal and be able to express $\mathrm{P} 12$ fate. Integration of Wnt signal to the lin-3/let-23 EGF signaling pathway may be at the level of LIN-3 signal, LET-23 receptor or egl-5 transcription. 
epistasis analysis suggests that the lin-3/let-23 signaling pathway specifies P12 fate in the same fashion as it does for vulval induction. The same lin-3/let-23 pathway has also been shown to organize $C$. elegans male spicule development (Chamberlin and Sternberg, 1994). Thus a conserved EGF signaling pathway is used at different times and in different cell types during development to organize cell fate specification.

During $C$. elegans hermaphrodite vulval development, a signal from the anchor cell (AC) in the gonad is required to induce three AC-proximal vulval precursor cells (VPC) to take on vulval fate, whereas the three distal VPCs produce nonspecialized epidermis (reviewed by Horvitz and Sternberg, 1991). This AC signal is an EGF-like protein encoded by the gene lin-3 (Hill and Sternberg, 1992). lin-3 expression revealed by a lin-3-lac Z reporter is detected in the AC at proper time for vulval induction (Hill and Sternberg, 1992). In male spicule development, lin-3 mutants display defects similar to those observed in animals with $\mathrm{F}$ and $\mathrm{U}$ cells ablated (Chamberlin and Sternberg, 1993, 1994), suggesting that F and U cells may be the source of LIN-3 signal. The expression pattern of lin-3 has not been precisely determined since the genomic clone used to determine AC expression does not contain the entire coding region (J. Liu, P. Tzou, R. Hill, and P. Sternberg, unpublished). What is the LIN-3 signal source in the hermaphrodite tail region for $\mathrm{P} 12$ fate specification? By analogy to vulva and spicule development, the LIN-3 signal source should be posterior to the P11 and P12 cells to promote the posterior fate, P12. Transgenic animals bearing a lin-3-lacZ reporter gene with additional genomic sequence have been generated (R. Hill, C. Chang and P. Sternberg, unpublished observation). Indeed we found that lin-3-lacZ is expressed in the hermaphrodite tail region in newly hatched larvae, in the cells $\mathrm{K}, \mathrm{K}^{\prime}$, and the daughters of $\mathrm{K}$, just posterior to the P11/P12 cells. To verify that the reporter gene expression truly reflects LIN-3 activity, we need to identify the source of the inductive signal for the P12 fate. Ablation experiments have been conducted to identify the source (Sulston and White, 1980; R. Hill, personal communication; L. Jiang, unpublished observation). We eliminated cells, including repD, K, K', F and $\mathrm{U}$, or combinations of these cells, in the rectal region by a laser microbeam to examine if the P12 fate would be disrupted. As the cells in the rectal region are essential for animal survival and growth, and the development of animals with ablated cells arrests before we could score the cell fates, such ablation attempts have been unsuccessful.

\section{HOM-C gene egl-5 is regulated by the EGF signaling pathway}

HOM-C genes function in multiple cell types and at multiple times during development. Their expression patterns are finely tuned by diverse intercellular and intracellular interactions, which are important for the functions of HOM-C genes. $C$. elegans HOM-C genes, lin-39, mab-5 and egl-5, are turned on early during embryogenesis in a position-specific manner from anterior to posterior along body axis (Wang et al., 1993). Their dynamic expression patterns and the regulation of their expression patterns have not been studied in detail until recently (Harris et al., 1996; Salser and Kenyon, 1996; Maloof and Kenyon, 1998). Here we have studied the interaction between the HOM-C gene, egl-5, and the lin-3/let-23 EGF signaling pathway in the specification of $\mathrm{P} 11 / \mathrm{P} 12$ cell fates and demonstrated that egl-5 is a downstream target of the lin-3/let23 intercellular signaling pathway in specifying the P12 fate. egl-5 expression is turned on in P12 lineage upon activation of the lin-3/let-23 pathway, and it is necessary for P12 fate specification.

Interactions between HOM-C genes and the lin-3/let-23 pathway have also been examined in $C$. elegans vulval development. Genetic analysis revealed two functions of lin39 during vulval development (Clark et al., 1993; Clandinin et al., 1997; Maloof and Kenyon, 1998): early LIN-39 activity specifies VPCs by creating a permissive condition for vulval differentiation; later LIN-39 activity regulates the response of VPCs to the lin-3/let-23 pathway in the specification of a subset of VPCs as vulval tissue. Epistasis analysis has suggested that for the late role LIN-39 either regulates essential effectors of the response to inductive signal or itself is a target of the lin-3/let-23 signaling pathway. Our molecular genetic analysis provides evidence that in the case of P12 fate specification, the HOM-C gene egl-5 is a downstream target of the lin-3/let-23 inductive pathway. If lin-39 is indeed a target of the lin-3/let-23 pathway during vulval development, as the data of Clandinin et al. (1997) and Maloof and Kenyon (1998) suggest, HOM-C genes may thus provide cell-specific responses to the same signaling pathway by being distinct targets.

\section{lin-44 and lin-3 act synergistically}

Interactions between Wnt and growth signaling pathways can be either synergistic or antagonistic. Co-operative interactions between Wnt genes and members of the fibroblast growth factor (FGF) family have been identified during Xenopus embryonic development (Christian et al., 1992) and mammalian oncogenesis (Shackleford et al., 1993; Pan et al., 1995). Recently antagonistic interactions between wingless and EGFR signaling pathways have also been demonstrated during Drosophila larval cutical patterning (Szüts et al., 1997). Here we observed synergistic interactions between a Wnt protein, LIN-44, and the EGF-like protein LIN-3 in C. elegans P12 neuroectoblast fate specification. Moreover, this synergistic interaction also exists between the hypothesized Wnt receptor LIN-17 and LIN-3.

How do these two signaling pathways function in concert to specify P12 fate? We favor the model that both pathways are required for proper P12 fate specification and they act at different developmental times. First, LIN-3 overexpression experiments indicate that LIN-3 signal is required in early L1 before P11/P12 enter the ventral cord to induce P12 fate. Second, lin-44 expression is turned on during embryogenesis, much earlier than the time of P11/P12 induction (Herman et al., 1995). Third, the unique effect of overexpression of LIN3EGF during late embryogenesis in lin-44 mutant suggests that lin-44 function may be important in the early phase of P12 fate specification. It is possible that the Wnt pathway regulates the competence of the cells to respond to the LIN-3 inductive signal. But the Wnt signal alone is not sufficient to promote P12 fate, as overexpression of LIN-44 in wild-type animals has no effect on P11/P12 cell fate specification (L. Jiang and M. Herman, unpublished). These two pathways may also interact at multiple levels, for example, the Wnt pathway can regulate both the signaling cell and the responding cell. We set out to test the simple possibility that lin- 44 affects P12 fate by 
regulating lin-3 expression in the tail region. Results from three sets of experiments do not support this hypothesis. First, overexpression of LIN-3EGF can not completely bypass the requirement for lin-44 function. A lin-44 mutant decreased the response of P11 cell to LIN-3EGF overexpression (Fig. 2A,B). In addition, overexpression of LIN-3EGF during late embryogenesis caused a unique reverse cell fate transformation in a lin-44 mutant background (Fig. 2B). Second, lin-44 mutation did not affect lin-3 expression as revealed by a lin-3$l a c Z$ reporter gene. Third, decrease of lin-3 activity had a more severe effect on P11/P12 fates than decrease of lin-44 activity in a sensitized genetic background. Although our data do not favor this simple hypothesis, we can not completely rule out the possibility that lin-44 somehow affects the property of the lin-3 expressing cell in the tail region or the Wnt pathway may regulate LIN-3 protein processing. Further elucidation of how and where these two signaling pathways intersect requires additional genetic and molecular studies of both pathways.

\section{Temporal regulation of gene interactions}

Temporal control of gene activity is crucial for proper gene function during development. During our study of P12 neuroectoblast fate specification, we observed an unexpected phenomenon, in that overexpression of the same protein can result in opposite P11/P12 cell fate transformation depending on the time of induction. When overexpression of EGL-5 is induced in newly hatched larvae, it causes a P12 to P11 fate transformation (Fig. 2C), which contradicts its role in specifying P12 fate. This observation leads us to propose that the timing of egl-5 expression is critical for P12 fate specification. To specify P12 fate, egl-5 expression has to be turned on around the time of the first division of P11/P12 cells. If egl-5 expression is turned on earlier, P12 fate would not be specified correctly. Consistent with this, in wild-type animals egl-5-lacZ expression is not detected in P12 cell at the early L1 stage (Wang et al., 1993). Similar ON-OFF behavior occurs in the activity of another HOM-C gene, mab-5 (Salser and Kenyon, 1996). mab-5 expression switching on and off in a lineage has been demonstrated to be important to control cell proliferation, differentiation and morphogenesis during development (Salser and Kenyon, 1996). In the case of LIN-3, when overexpression of LIN-3EGF is induced during late embryogenesis in a lin-44(n1792) background, it potentiates the P12 to P11 fate transformation caused by a lin-44 mutation (Fig. 2B). This effect depends on both overexpression of LIN3EGF and lack of LIN-44 activity. As activation of the lin3/let-23 pathway can turn on egl-5 expression at the time of P12 fate induction, it might be that lack of LIN-44 activity enables egl-5 expression to be turned on by LIN-3EGF earlier than usual, thus disrupting P12 fate specification. The timing difference in these two cases is consistent with this hypothesis as the effect of LIN-3 overexpression is earlier than that of EGL-5 overexpression. Another example of the bi-directional $\mathrm{P} 11 / \mathrm{P} 12$ fate transformation is found in mab-5 mutants (Kenyon, 1986). Loss-of-function mutants of mab-5 show P11 to P12 as well as P12 to P11 fate transformations. As mutual repression has been suggested between HOM-C genes (Salser and Kenyon, 1993), temporal and spatial expression of egl-5 might be abnormal in mab-5 mutants, therefore resulting in cell fate transformation in both directions. A direct test of this hypothesis will require detailed temporal and spatial analysis of egl-5 expression pattern in different genetic backgrounds.

\section{Model for P12 fate specification}

We propose the following model for P12 neuroectoblast fate specification (Fig. 4). In newly hatched larvae, LIN-44 signal acts via receptor LIN-17 to set up the competence of P11 and P12 cells to respond to the inductive signal and be able to express P12 fate. egl-5 expression is kept off in both P11 and P12 cells. Later, an inductive signal LIN-3 coming from the posterior region activates LET-23 receptor activity in the posterior cell of the P11/P12 pair. Activation of the lin-3/let23 pathway turns on egl-5 expression, which specifies the posterior cell to take on $\mathrm{P} 12$ fate. lin-15 negatively regulates let-23 activity and prevents the anterior cell becoming P12. Information from the Wnt signaling pathway may be integrated into the lin-3/let-23 EGF signaling pathway at the level of LIN3 signal, LET-23 receptor or egl-5 transcription. Thus the temporal and spatial co-ordination and interactions between the Wnt signal, EGF signal and HOM-C transcription factor are important for P12 fate specification.

We are grateful to Craig Hunter for sending us the egl-5 cDNA clone and the muIs13 strain. We thank Michael Herman for the lin17(n671) lin-44(n1792) strain and for many stimulating discussions. We thank Michael Herman, Howard Lipshitz, Kai Zinn, Tom Clandinin, Maureen Barr, Jing Liu, Minqin Wang, Peter Becker, Marie-Anne Félix, Giovanni Lesa, Aidyl Gonzalez-Serrichio, Chris Lacenere, René Garcia and Bhagwati Gupta for comments on the manuscript. This work was supported by a USPHS grant HD23690 to P. W. S. and by the HHMI, with which P. W. S. is an investigator.

\section{REFERENCES}

Aroian, R. V., Koga, M., Mendel, J. E., Ohshima, Y. and Sternberg, P. W. (1990). The let-23 gene necessary for Caenorhabditis elegans vulval induction encodes a tyrosine kinase of the EGF receptor subfamily. Nature 348, 693-699.

Aroian, R. V. and Sternberg, P. W. (1991). Multiple functions of let-23, a Caenorhabditis elegans receptor tyrosine kinase gene required for vulval induction. Genetics 128, 251-277.

Aroian, R. V., Lesa, G. M. and Sternberg, P. W. (1994). Mutations in the Caenorhabditis elegans let-23 EGFR-like gene define elements important for cell-type specificity and function. EMBO J. 13, 360-366.

Brenner, S. (1974). The genetics of Caenorhaebditis elegans. Genetics 77, 7194.

Chamberlin, H. M. and Sternberg, P. W. (1993). Multiple cell interactions are required for fate specification during male spicule development in Caenorhabditis elegans. Development 118, 297-324.

Chamberlin, H. M. and Sternberg, P. W. (1994). The lin-3/let-23 pathway mediates inductive signaling during male spicule development in Caenorhabditis elegans. Development 120, 2713-2721.

Chisholm, A. (1991). Control of cell fate in the tail region of C. elegans by the gene egl-5. Genes Dev. 111, 921-932

Christian, J. L., Olson, D. J. and Moon, R. T. (1992). Xwnt-8 modifies the character of mesoderm induced by bFGF in isolated Xenopus ectoderm. EMBO J. 11, 33-41.

Clandinin, T. R., Katz, W. S. and Sternberg, P. W. (1997). Caenorhabditis elegans HOM-C genes regulate the response of vulval precursor cells to inductive signal. Devel. Biol. 182, 150-161.

Clark, S. G., Stern, M. J. and Horvitz, H. R. (1992a). C. elegans cellsignaling gene sem-5 encodes a protein with $\mathrm{SH} 2$ and $\mathrm{SH} 3$ domains. Nature 356, 340-344.

Clark, S. G., Stern, M. J. and Horvitz, H. R. (1992b). Genes involved in two Caenorhabditis elegans cell-signaling pathways. Cold Spring Harbor Symp. Quant. Biol. 57, 363-373.

Clark, S. G., Chisholm, A. D. and Horvitz, H. R. (1993). Control of cell 
fates in the central body region of C. elegans by the homeobox gene lin-39. Cell 74, 43-55

Clark, S. G., Lu, X. W. and Horvitz, H. R. (1994). The Caenorhabditis elegans locus lin-15, a negative regulator of a tyrosine kinase signaling pathway, encodes 2 different proteins. Genetics 137, 987-997.

Fantl, W. J., Johnson, D. E. and Williams, L. T. (1993). Signaling by receptor tyrosine kinases. Ann. R. Bioch. 62, 453-481.

Fire, A., Harrison, S. W. and Dixon, D. (1990). A modular set of lacZ fusion vectors for studying gene expression in Caenorhabditis elegans. Genetics 93, 189-198.

Ferguson, E. L. and Horvitz, H. R. (1985). Identification and characterization of 22 genes that affect the vulval cell lineages of the nematode Caenorhabditis elegans. Genetics 110, 17-72.

Ferguson, E. L., Sternberg, P. W. and Horvitz, H. R. (1987). A genetic pathway for the specification of the vulval cell lineages of Caenorhabditis elegans. Nature 326, 259-267.

Fixsen, W., Sternberg, P. W., Ellis, H. and Horvitz, H. R. (1985). Genes that affect cell fates during the development of Caenorhabditis elegans. Cold Spring Harbor Symp. Quant. Biol. 50, 99-104.

Han, M. and Sternberg, P. W. (1990). let-60, a gene that specifies cell fates during C. elegans vulval induction, encodes a RAS protein. Cell 63, 921-931.

Harris, J., Honigberg, L., Robinson, N. and Kenyon, C. J. (1996). Neuronal cell-migration in Caenorhabditis elegans - regulation of hox geneexpression and cell position. Development 122, 3117-3131.

Herman, M. A. and Horvitz, H. R. (1994). The Caenorhabditis elegans gene lin-44 controls the polarity of asymmetric cell divisions. Development 120, 1035-1047.

Herman, M. A., Vassilieva, L. L., Horvitz, H. R., Shaw, J. E. and Herman, R. K. (1995). The C. elegans gene lin-44, which controls the polarity of certain asymmetric cell divisions, encodes a Wnt protein and acts cell nonautonomously. Cell 83, 101-110.

Herman, R. K. and Hedgecock, E. M. (1990). Limitation of the size of the vulval primordium of Caenorhabditis elegans by lin-15 expression in surrounding hypodermis. Nature 348, 169-171.

Hill, R. J. and Sternberg, P. W. (1992). The gene lin-3 encodes an inductive signal for vulval development in C. elegans. Nature 358, 470-476.

Horvitz, H. R. and Sternberg, P. W. (1991). Multiple intercellular signaling systems control the development of the Caenorhabditis elegans vulva. Nature 351, 535-541.

Huang, L. S., Tzou, P. and Sternberg, P. W. (1994). The lin-15 locus encodes two negative regulators of Caenorhabditis elegans vulval development. Molec. Biol. Cell. 5, 395-411.

Hunter, C. P. and Kenyon, C. (1995). Specification of anteroposterior cell fates in Caenorhabditis elegans by Drosophila Hox proteins. Nature 377, 229-232.

Immergluck, K., Lawrence, P.A. and Bienz, M. (1990). Induction across germ layers in Drosophila mediated by a genetic cascade. Cell 62, 261-268

Katz, W. S., Hill, R. J., Clandinin, T. R. and Sternberg, P. W. (1995) Different levels of the $C$. elegans growth-factor lin-3 promote distinct vulval precursor fates. Cell 82, 297-307.

Kenyon, C. (1986). A gene involved in the development of the posterior body region of Caenorhabditis elegans. Cell 46, 477-487.

Kornfeld, K. (1997). Vulval development in Caenorhabditis elegans. Trends Genet. 13, 55-61.

Krumlauf, R. (1994). Hox genes in vertebrate development. Cell 78, 191-201.

Lawrence, P. A. and Morata, G. (1994). Homeobox genes, their function in Drosophila segmentation and pattern formation. Cell 78, 181-189.

Lesa, G. M. and Sternberg, P. W. (1997). Positive and negative tissue-specific signaling by a nematode epidermal growth factor receptor. Mol. Biol. Cell 8, 779-793.

Lewis, E. B. (1978). A gene complex controlling segmentation in Drosophila. Nature 276, 565-570.

Maloof, J. N. and Kenyon, C. (1998). The hox gene lin-39 is reuired during C. elegans vulval induction to select the outcome of Ras signaling. Development 125, 181-190.
Mastick, G. S., Mckay, R., Oligino, T., Donovan, K. and Lopez, A. J. (1995). Identification of target genes regulated by homeotic proteins in Drosophila melanogaster through genetic selection of Ultrabithorax protein-binding sites in yeast. Genetics 139, 349-363.

Mello, C. C., Kramer, J. M., Stinchcomb, D. and Ambros, V. (1991). Efficient gene transfer in C. elegans - extrachromosomal maintenance and integration of transforming sequences. EMBO J. 10, 3959-3970.

Moon, R. T., Brown, J. D. and Torres, M. (1997). Wnts modulate cell fate and behavior during vertebrate development. Trends Genet. 13, 157-162.

Nusse, R. and Varmus, H. E. (1992). Wnt genes. Cell 69, 1073-1087.

Pan, M. G., Wang, Y. H., Hirsch, D. D., Labudda, K. and Stork, P. J. (1995). The Wnt-1 proto-oncogene regulates MAP kinase activation by multiple growth factors in PC12 cells. Oncogene 11, 2005-2012.

Russnak, R. H. and Candido, E. P. M. (1985). Locus encoding a family of small heat-shock genes in Caenorhabditis elegans -2 genes duplicated to form a 3.8-kilobase inverted repeat. Molec. Cell. Biol. 5, 1268-1278.

Salser, S. J. and Kenyon, C. (1992). Activation of a C. elegans Antennapedia homolog in migrating cells controls their direction of migration. Nature $\mathbf{3 5 5}$, 255-258.

Salser, S. J., Loer, C. M. and Kenyon, C. (1993). Multiple HOM-C gene interactions specify cell fates in the nematode central-nervous-system. Genes Dev. 7, 1714-1724.

Salser, S. J. and Kenyon, C. (1994). Patterning in C. elegans: homeotic cluster genes, cell fates and cell migrations. Trends Genet. 10, 159-164.

Salser, S. J. and Kenyon, C. (1996). A C. elegans hox gene switches on, off, on and off again to regulate proliferation, differentiation and morphogenesis. Development 122, 1651-1661.

Sawa, H., Lobel, L. and Horvitz, H. R. (1996). The Caenorhabditis elegans gene lin-17, which is required for certain asymmetric cell divisions, encodes a putative protein similar to seven-transmembrane protein similar to the Drosophila Frizzle protein. Genes Dev. 10, 2189-2197.

Shackleford, G. M., Willert, K., Wang, J. W. and Varmus, H. E. (1993) The Wnt-1 protooncogene induces changes in morphology, gene-expression, and growth-factor responsiveness in PC12 cells. Neuron 11, 865-875.

Sternberg, P. W., Hill, R. J., Jongeward, G., Huang, L. S. and Carta, L. (1992). Intercellular signaling during Caenorhabditis elegans vulval induction. Cold Spring Harbor Symp. Quant. Biol. 57, 353-362.

Sternberg, P. W. and Horvitz, H. R. (1988). lin-17 mutations of Caenorhabditis elegans disrupt certain asymmetric cell divisions. Dev. Biol. 130, 67-73.

Stringham, E. G., Dixon, D. K., Jones, D. and Candido, E. P. M. (1992). Temporal and spatial expression patterns of the small heat-shock (hsp-16) genes in transgenic Caenorhabditis elegans. Molec. Biol. Cell 3, 221-233.

Sulston, J. E. and Horvitz, H. R. (1977). Postembryonic cell lineages of the nematode Caenorhabditis elegans. Dev. Biol. 56, 110-156.

Sulston, J. E. and White, J. G. (1980). Regulation and cell autonomy during postembryonic development of Caenorhabditis elegans. Dev. Biol. 78, 577597.

Sulston, J. E., Schierenberg, E., White, J. G. and Thomson, J. N. (1983). The embryonic-cell lineage of the nematode Caenorhabditis elegans. Dev. Biol. 100, 64-119.

Sundaram, M. and Han, M. (1996). Control and integration of cell signaling pathways during C. elegans vulval development. BioEssays 18, 473-480.

Szüts, D., Freeman, M. and Bienz, M. (1997). Antagonism between EGFR and Wingless signaling in the larval cutical of Drosophila. Development 124, 3209-3219.

van den Heuvel, M., Nusse, R., Johnson, P. and Lawrence, P. A. (1989) Distribution of wingless protein in Drosophila embryos: a protein involved in cell-cell communication. Cell 59, 739-749.

Vincent, J. P. and Lawrence, P. A. (1994). Developmental genetics - it takes three to distalize. Nature 372, 132-133.

Wang, B. B., Muller-Immergluck, M. M., Austin, J., Robinson, N. T., Chisholm, A. and Kenyon, C. (1993). A homeotic gene cluster patterns the anteroposterior body axis of C. elegans. Cell 74, 29-42. 